\title{
Data Anonymization Using Imbalanced Data for Deep Learning with Uppersampling and Undersampling
}

\author{
Ayahiko Niimi \\ Faculty of Systems Information Science, \\ Future University Hakodate, Japan
}

\begin{abstract}
Most real data are imbalanced and thus present difficulties as a subject of classification problems. Some research has been conducted on the topic of imbalanced data. However, managing such data in deep learning has not been considered. In this study, we propose privacy-protection data mining using imbalanced data through deep learning. We discuss existing privacy-protection data mining, study its features, and examine a deep learning based anonymizing tool. Experiments using various anonymization tools confirm that deep learning does not reduce data accuracy by making that data anonymous. Under and uppersampling are both used for imbalanced data, but the tendencies of these approaches to reduce data accuracy because of anonymization have not been confirmed.
\end{abstract}

\section{Introduction}

Big data and data mining both involve large amounts of data. However, the data in question may contain personal and private information. It is critical to consider how the data may be used and then apply the appropriate risk assessment method. Privacypreserving data mining is therefore used to employ data, safely, so that private information is properly secured.

By contrast, deep learning (which is a new technology that has attracted considerable attention e.g., in AlphaGo [1] and image analysis) is defined as a machine learning method. Most real data are imbalanced and thus present difficulties as a subject of classification problems. When the amount of data for a positive class is sufficiently greater than those for a negative class, it is expressed as TP $\gg \mathrm{TN}$, where the effect of $\mathrm{TN}$ on the rate of correct answers is low. Many datasets are characterized by very few specific classes of data, such as fraud detection, abnormality detection, and multi-class classification. While some studies have been conducted on imbalanced data [2], [3], managing imbalanced data in deep learning has not yet been considered. We have previously determined the deep learning processes required for solving these problems and we proposed appropriate methods for applying deep learning [4], [5].
In this study, we propose privacy-preserving data mining through deep learning. We consider the features of and apply existing privacy-preserving data mining techniques, while examining anonymization tools for deep learning.

\section{Privacy-Preserving Data Mining}

Big data and data mining both onvolve large amounts of data. However, the data in question may contain personal and private information. It is critical to consider how the data may be used and then apply the appropriate risk assessment method. Privacypreserving data mining is used to employ data safely in such a manner that personal and private information are secured [6]-[8]. Privacy-preserving data mining is a generic term describing techniques that extract useful information from data while protecting privacy.

Privacy-preserving data mining involves techniques related to input data, secret computation, and output data. Research on input data involves privacy protection technology. This technology is used when transforming raw data into data that can be analyzed. Input-data privacy-preserving technology reduces the risk of privacy infringement by processing or deleting data that may expose individuals to identification. Representative methods include "anonymization" and "randomization." Secret computation enables the analysis of results only, while concealing the data. This technique involves the accumulation of data in a state that is nonsensical or illogical on a standalone basis and analyzes the data simply by displaying it. Secret computation is based on techniques such as encryption and secret sharing. We can identify the administrator of anonymized data and the person performing the data mining task, through confidential calculations [9]. Technology related to output data involves reducing the risk of privacy infringement when outputting the results of analyzed data. This technique avoids the risk of exposing the private attributes of an individual by controlling responses to queries. This involves analyzing and adding noise to the query results.

\subsection{Input Data}

We first consider the "anonymization" and "randomization" of representative methods of input- 
data privacy-protection technology. Anonymization prevents data from being returned to an individual in a raw form. Anonymization has the properties of attribute deletion, attribute change, and generalization: - attribute deletion refers to the act of deleting a specific attribute; attribute change involves securing an individual's identity by replacing private information with other information (i.e., information that can be revealed without violating privacy); and generalization is a technique to reduce specificity and discrimination by increasing the granularity of information.

The sole application of these methods is insufficient to reduce the risk of privacy infringement. For example, the individual can often be identified when the amount of data is large, when linking with other information, or when the data are accompanied by time changes.

The indices "k-Anonymity" and "l-diversity" have been proposed as means of evaluating the risk of respecifying anonymized data and estimating attributes [10], [11].

The index k-Anonymity reduces the risk of individuals being identified by making $\mathrm{k}$ or more pieces of information unidentifiable. For example, when $\mathrm{k}=3$, data are processed such that three or more pieces of data exist that have attribute values for identifying the same individual. Thus, even if a single attribute value identifying an individual is viewed due to the presence of three or more attributes, identifying an individual becomes impossible.

With k-Anonymity, individual attributes can be estimated if their attributes are biased, even if $\mathrm{k}$ data of the same kind exists. The index l-diversity can help reduce the risk of privacy infringement through individual attribute estimation.

Randomization reduces the risk of individual identification by randomizing or adding noise based on rules predefined for raw data. Because data are converted to "nonexistent data," randomization makes associating data with individuals difficult, and the results of data mining are thus affected.

A method of restoring original data through data randomization using Bayesian estimation has been proposed [7].

\subsection{Multi-party Computation}

Multi-party computation is a generic term to describe a method of outputting only the operation result while hiding information related to each party among multiple analysts [9]. This method encrypts input values, produces only calculation results, and employs cryptograms having homomorphism. If these cryptograms are used, performing computations while hiding confidential information by encrypting it is possible. This method decodes only the calculation results.

\subsection{Output Data}

If the data being considered contain a bias or are small, in quantity, it is possible to specify the individual by a search. Techniques such as limiting and adding noise to the output results are employed to address this potential threat to privacy. This kind of technique is called a query inference control.

\section{Deep Learning}

Deep learning is a new technology that has recently attracted considerable attention in the field of machine learning [12]- [14]. Numerous studies have focused on the basic theory behind and methods of improving deep learning [14]-[16].

Apache Spark is a platform that processes large amounts of data by generalizing map/reduce processing. Specifically, it processes data by caching the work memory. Efficient iterative algorithms are executed by maintaining shared data, which is used for repeated processing in the memory. In addition, machine learning and graph algorithm libraries are prepared, and an environment for stream data mining can be easily built.

$\mathrm{H} 2 \mathrm{O}$ is a library of deep learning for Spark. SparkR is an $\mathrm{R}$ package that provides a light weight frontend for Apache Spark from R. Similar to the R dataframe package, dplyr, but applicable to large datasets, SparkR (Spark 1.5.0) provides distributed dataframe implementation that supports operations such as selection, filtering, and aggregation. SparkR also supports distributed machine learning using MLlib.

In this study, we use $\mathrm{R}$ and Spark. An extensive library can be used with $\mathrm{R}$ to achieve high performance through parallel and distributed processing of Spark.

\section{Imbalanced Data}

Many datasets are imbalanced, meaning that they are biased in terms of the amount of data given for each class. Data bias for each class negatively affects classification learning. In classification learning, accurasy is often necessary for classification evaluation. For example, assume that a classification result is the confusion matrix shown in Table I.

Table I. Confusion Matrix of Imbalanced Data

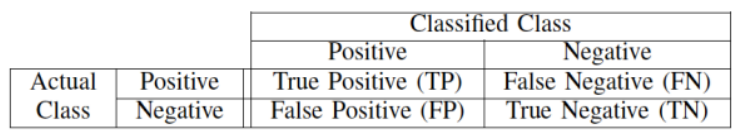

Accurasy is then defined as follows.

Accuracy $=(\mathrm{TP}+\mathrm{TN})=(\mathrm{TP}+\mathrm{FP}+\mathrm{TN}+\mathrm{FN})(1)$ 
When the amount of data of a positive class is sufficiently greater than that of a negative class, it is expressed as TP $\gg \mathrm{TN}$, where the effect of $\mathrm{TN}$ on the rate of correct answers is small.

Many datasets have very few specific classes of data, such as fraud detection, abnormality detection, and multi-class classification. One of the objectives of this research is to confirm whether privacy-protection mining is effective with such data.

In general, for heterogeneous data, undersampling to reduce majority class data and uppersampling to increase minority class data are performed.

UnderSampling is a method to equalize the number of data of each class by reducing the data of the majority class of imbalanced data. The work of Wallance et al. Shows that UnderSampling works well for various imbalanced data sets [17]. However, if the data difference between each class is large, it is necessary to reduce the data of many major classes, so there is a possibility that the feature quantity of the data may be insufficient.

OverSampling is a method to equalize the number of data of each class by increasing the data of the minority class of imbalanced data. The easiest way to increase the amount of data is to randomly select and copy data of the minority class. However, with this method, there is a possibility that overfitting may occur during learning, resulting in a learner with low generalization performance, because there are multiple identical data. Therefore, methods that do not increase the same data, such as SMOTE [18], which is the method proposed by Chawla et al., Have been proposed.

Chawla et al. Propose an oversampling method called SMOTE (Synthetic Minority Over-Sampling Technique) [18]. SMOTE is a method to generate new data by combining data. By doing so, data can be generated in the area where there is no data, so we can expect improvement in the performance of the learning device. As a result of performance comparison using ROC curve, the improvement of performance was confirmed.

Wallace et al. Changed the number of attributes, the number of training data, the ratio of data in the minority class, etc., and conducted experiments to identify the conditions under which the imbalanced data problem worsens [17]. In experiments with the number of attributes, it was found that SMOTE and weighted-SVM are effective when the number of attributes is small, but the performance decreases as the number of attributes increases. In the experiment of the number of training data, it was found that SMOTE and weighted-SVM are more effective as the number of data is larger and the ratio of data of minority class is larger. In addition, it was found that UnderSampling and Bagging were always effective in these experiments.
As described above, although there are several previous studies on imbalanced data, the imbalanced data problem for deep learning with privacy protection has not been studied.

\section{Privacy-Preserving Data Mining In Deep Learning}

We next consider privacy-protection data mining in deep learning. Deep learning calculations are intensive, and the accuracy is reduced if the data are randomized. With randomization, a reconstruction calculation is difficult. If it is a secret computation, the calculation is intensive. To manage a large amount of data, an appropriate method for anonymizing input data must be applied.

Many anonymizing tools have been developed. As opensource anonymizing tools, the Cornell Anonymization Toolkit (CAT) [19] and UTD Anonymization ToolBox (UAT) [20] are available.

The CAT is designed for interactively anonymizing published datasets to limit identification disclosure of records through various attacker models.

The UAT was developed by the University of Texas at Dallas Data Security and Privacy Lab. The toolbox currently contains the following six anonymization methods that are based on three privacy definitions:

1) Datafly;

2) Mondrian multidimensional k-Anonymity;

3) Incognito;

4) Incognito with 1-diversity;

5) Incognito with t-closeness; and

6) Anatomy.

Many anonymization tools have been developed, but they are not specific to deep learning. Deep learning has the following features:

1) high expressive power of the learning model;

2) uses extensive input data; and

3) performs iterative calculations.

Existing anonymizing tools, such as association rules and decision trees, have been devised for machine learning, where the expressive power of learning models is low. Because deep learning has a high expressive power of models, individuals may be identified even after they employ input-data privacy rotection technology. In addition, since deep learning is applicable to extensive data, the calculation time must also be emphasized.

After considering the problems of existing anonymizing tools, we must consider an anonymizing tool for deep learning. An anonymization tool for deep learning must have the following attributes: an individual cannot be identified through deep learning, and the number of calculations must be small. In addition, as we assume that the existing deep learning framework performs parallel distributed processing, 
the anonymizing tool should ideally perform parallel distributed processing.

\section{Dataset}

A sample data from the UAT was used to compile a dataset for experiments in this study. The task for this dataset is to predict whether income exceeds $\$ 50 \mathrm{~K} / \mathrm{yr}$ based on census data. Thus, this dataset is also known as the "Census-Income" or "Adult" dataset.

\section{Experiment 1}

To investigate the problem of using an existing anonymizing tool for deep learning, we conducted an experiment in which deep learning was employed to anonymize data using UAT as the anonymization tool. UAT is an open-source anonymization tool and is thus easy to use it in experiments. Apache Spark and Sparking Water were used as deep learning frameworks on $\mathrm{R}$.

The experimental environment was conducted on AWS EC2. The instance type was an m4.large, 2.3 GHz Intel Xeon E5-2686 v4 (Broadwell) with 8 GiB of RAM.

Sample data from UAT (census-income 1K.data and census-income ALL.data) were used. We used census-income 1K.data (42 attributes, 463 cases) as the training data and census-income ALL.data (42 attributes, 95130 cases) as the test data. As a parameter of UTA, $\mathrm{k}=10$ was used. Through anonymization using UAT, the number of attributes changed from 42 to 40 . The time required for anonymization was $8 \mathrm{~s}$ for census-income $1 \mathrm{~K}$.data and $34 \mathrm{~s}$ for census-income ALL.data. The middle layer of deep learning included three layers $(100,100$, and 200). The activation functions of deep learning are described as follows:

- RectifierWithDropout;

- Rectifier;

- TanhWithDropout;

- Tanh;

- MaxoutWithDropout; and

- Maxout.

The error rates for the test data of deep learning before and after anonymization are given in Table II.

TABLE II. Results of Deep Learning

\begin{tabular}{lrr}
\hline Activation function & $\begin{array}{r}\text { error rate } \\
\text { before anonymization }\end{array}$ & $\begin{array}{r}\text { error rate } \\
\text { after anonymization }\end{array}$ \\
\hline \hline RectifierWithDropout & 5.74 & 5.74 \\
\hline Rectifier & 6.19 & 6.19 \\
\hline TanhWithDropout & 7.20 & 7.20 \\
\hline Tanh & 7.73 & 7.73 \\
\hline MaxoutWithDropout & 8.22 & 8.22 \\
\hline Maxout & 9.5 & 9.5 \\
\hline
\end{tabular}

As a result of anonymization, no decline in accuracy was observed.

\section{Experiment 2}

Table III shows the amount of data used in Experiment 1 for each data class.

TABLE III. Class Distribution (Original)

\begin{tabular}{lll}
\hline filename & - _50000 & $50000+$ \\
\hline \hline census-income_1K.data & 433 & 30 \\
census-income_ALL.data & 89651 & 5479 \\
\hline
\end{tabular}

In contrast to Experiment 1 that involved only a single experiment, Experiment 2 involved a set of experiments in which we changed the class distribution of data used for learning through underand uppersampling (Tables IV and VI.) The parameters of deep learning were the same as in Experiment 1, and RectifierWithDropout was used as the activation function for deep learning. Tables $\mathrm{V}$ and VII show the results.

With the dataset used in the experiment, minority class data less undersampling was ineffective. Uppersampling, which increases the amount of minority data, improved data accuracy. With uppersampling, because the same data were copied, we assumed the anonymization process would be affected, however, there was no tendency in which the precision was diminished as a result of anonymization.

TABLE IV. Class Distribution (Undersampling)

\begin{tabular}{lll}
\hline filename & - _50000 & $50000+$ \\
\hline \hline census-income_30.data & 30 & 30 \\
census-income_60.data & 60 & 30 \\
census-income_90.data & 90 & 30 \\
census-income_120.data & 120 & 30 \\
census-income_150.data & 150 & 30 \\
census-income_180.data & 180 & 30 \\
census-income_210.data & 210 & 30 \\
census-income_240.data & 240 & 30 \\
census-income_270.data & 270 & 30 \\
census-income_300.data & 300 & 30 \\
census-income_330.data & 330 & 30 \\
census-income_360.data & 360 & 30 \\
census-income_390.data & 390 & 30 \\
census-income_420.data & 420 & 30 \\
census-income_1K.data & 433 & 30 \\
\hline
\end{tabular}


TABLE V. Results of Deep Learning (Undersampling)

\begin{tabular}{lrr}
\hline filename & $\begin{array}{r}\text { error rate } \\
\text { before } \\
\text { anonymization }\end{array}$ & $\begin{array}{r}\text { error rate } \\
\text { after } \\
\text { anonymization }\end{array}$ \\
\hline \hline census-income_30.data & 20.09 & 24.63 \\
census-income_60.data & 10.14 & 12.14 \\
census-income_90.data & 7.84 & 7.90 \\
census-income_120.data & 7.15 & 7.52 \\
census-income_150.data & 7.54 & 7.61 \\
census-income_180.data & 7.34 & 7.28 \\
census-income_210.data & 6.89 & 7.50 \\
census-income_240.data & 6.82 & 6.68 \\
census-income_270.data & 7.44 & 6.01 \\
census-income_300.data & 6.81 & 6.97 \\
census-income_330.data & 6.57 & 7.01 \\
census-income_360.data & 6.93 & 6.89 \\
census-income_390.data & 6.50 & 6.75 \\
census-income_420.data & 6.47 & 6.33 \\
census-income_1K.data & 5.74 & 5.74 \\
\hline
\end{tabular}

TABLE VI. Class Distribution (Uppersampling)

\begin{tabular}{lll}
\hline filename & - _50000 & $50000+$ \\
\hline \hline census-income_30u.data & 30 & 30 \\
census-income_60u.data & 60 & 60 \\
census-income_90u.data & 90 & 90 \\
census-income_120u.data & 120 & 120 \\
census-income_150u.data & 150 & 150 \\
census-income_180u.data & 180 & 180 \\
census-income_210u.data & 210 & 210 \\
census-income_240u.data & 240 & 240 \\
census-income_270u.data & 270 & 270 \\
census-income_300u.data & 300 & 300 \\
census-income_330u.data & 330 & 330 \\
census-income_360u.data & 360 & 360 \\
census-income_390u.data & 390 & 390 \\
census-income_420u.data & 420 & 420 \\
census-income_1K.data & 433 & 30 \\
\hline
\end{tabular}

TABLE VII. Results of Deep Learning (Uppersampling)

\begin{tabular}{lrr}
\hline filename & $\begin{array}{r}\text { error rate } \\
\text { before } \\
\text { anonymization }\end{array}$ & $\begin{array}{r}\text { error rate } \\
\text { after } \\
\text { anonymization }\end{array}$ \\
\hline \hline census-income_30u.data & 19.13 & 22.13 \\
census-income_60u.data & 8.63 & 9.90 \\
census-income_90u.data & 7.03 & 6.62 \\
census-income_120u.data & 6.26 & 6.31 \\
census-income_150u.data & 6.18 & 6.49 \\
census-income_180u.data & 6.25 & 6.48 \\
census-income_210u.data & 6.00 & 6.06 \\
census-income_240u.data & 5.75 & 5.58 \\
census-income_270u.data & 5.85 & 5.76 \\
census-income_300u.data & 5.62 & 5.60 \\
census-income_330u.data & 5.73 & 5.63 \\
census-income_360u.data & 5.74 & 5.50 \\
census-income_390u.data & 5.53 & 5.75 \\
census-income_420u.data & 5.62 & 5.58 \\
census-income_1K.data & 5.74 & 5.74 \\
\hline
\end{tabular}

\section{Conclusion}

In this study, we proposed privacy-protection data mining through deep learning. We discussed existing privacy protection data mining techniques, examined their features, and assessed an anonymizing tool for deep learning. Experiments using anonymization tools confirmed that deep learning does not reduce accuracy when anonymization is applied. Under and uppersampling were used for imbalanced data, but these techniques did not confirm the tendency of anonymization to reduce accuracy.

In a future study, we will conduct experiments using other types of datasets and examine problems encountered when using existing anonymization tools for deep learning.

\section{References}

[1] AlphaGo - DeepMind. (Access Date: 24 February, 2018). [Online]. Available: https://deepmind.com/research/alphago/

[2] B. C. Wallace, K. Small, C. E. Brodley, and T. A. Trikalinos, "Class imbalance, redux," in 2011 IEEE 11th International Conference on Data Mining, Dec 2011, pp. 754-763.

[3] H. He and E. A. Garcia, "Learning from imbalanced data," IEEE Transactions on Knowledge and Data Engineering, vol. 21, no. 9, pp.1263-1284, Sept 2009.

[4] M. Lichman, "UCI machine learning repository," 2013, (Access Date: 16 July, 2016). [Online]. Available: http://archive.ics.uci.edu/ml

[5] T. J. OZAKI. (2015) Data scientist in ginza, tokyo. (Access Date: 16 July, 2016). [Online]. Available: http://tjo-en.hatenablog.com/

[6] R. Agrawal and R. Srikant, "Privacy-preserving data mining," SIGMOD Rec., vol. 29, no. 2, pp. 439450, May 2000. [Online]. Available: http://doi.acm.org/10.1145/335191.335438

[7] C. C. Aggarwal and P. S. Yu, A General Survey of Privacy-Preserving Data Mining Models and Algorithms. Boston, MA: Springer US, 2008, pp. 1152. [Online]. Available: https://doi.org/10. 1007/978-0-387-70992-5 2

[8] J. Sakuma and S. Kobayashi, "Privacy-preserving data mining," The Japanese Society for Artificial Intelligence, vol. 24, no. 2, pp. 283-294, mar 2009.

[9] R. Cramer, I. Damg ${ }^{\circ}$ ard, and J. B. Nielsen, "Multiparty computation from threshold homomorphic encryption," in Advances in Cryptology - EUROCRYPT 2001, B. Pfitzmann, 
Ed. Berlin, Heidelberg: Springer Berlin Heidelberg, 2001, pp. 280-300.

[10] A. Machanavajjhala, J. Gehrke, D. Kifer, and M. Venkitasubramaniam, "L-diversity: privacy beyond k-anonymity," in 22nd International Conference on Data Engineering (ICDE’06), April 2006, pp. 24-24.

[11] L. SWEENEY, "k-anonymity: A model for protecting privacy," International Journal of Uncertainty, Fuzziness and Knowledge-Based Systems, vol. 10, no. 05, pp. 557-570, 2002. [Online]. http://www.worldscientific.com/doi/abs/10.1142/S02 18488502001648

[12] Y. Bengio, "Learning deep architectures for AI," Found. Trends Mach. Learn., vol. 2, no. 1, pp. 1-127, Jan. 2009, (Access Date: 16 July, 2016). [Online]. Available: http://dx.doi.org/10.1561/2200000006

[13] Q. Le, "Building high-level features using large scale unsupervised learning," in Acoustics, Speech and Signal Processing (ICASSP), 2013 IEEE International Conference on, May 2013, pp. 85958598 .

[14] I. J. Goodfellow, D. Warde-Farley, M. Mirza, A. Courville, and Y. Bengio, "Maxout Networks," ArXiv e-prints, Feb. 2013.

[15] A. Niimi, "Deep learning for credit card data analysis," in World Congress on Internet Security (WorldCIS-2015), Dublin, Ireland, Oct. 2015, pp. 7377.

[16] A. Niimi, "Deep learning for real credit card data," in Fuzzy Systems and Data Mining III, Proceedings of FSDM 2017, Hualien, Taiwan, Nov. 2017, pp. 303-310.

[17] B. Wallace, K. Small, C. Brodley, and T. Trikalinos, Class imbalance, redux, 2011, pp. 754763.

[18] N. V. Chawla, K. W. Bowyer, L. O. Hall, and W. P. Kegelmeyer, "Smote: Synthetic minority oversampling technique," Journal of Artificial Intelligence Research, vol. 16, pp. 321-357, 2002.

[19] Cornell anonymization toolkit. (Access Date: 31 January, 2017). [Online]. Available: https://sourceforge.net/projects/anony-toolkit/

[20] Utd anonymization toolbox. (Access Date: 31 January, 2017). [Online]. Available: http://www.cs.utdallas.edu/dspl/cgibin/toolbox/index.php

\section{Acknowledgements}

This work was supported by JSPS KAKENHI Grant Number JP17K00310. 